\title{
Adsorption and Rejection of PAEs by Modified Nanofiltration Membrane
}

\author{
Xindong Li ${ }^{1, ~}$, Guozi Ouyang ${ }^{1}$, Tingting Tian ${ }^{1}$, Lijinhong Huang ${ }^{1,2}$, and Xin Zhang ${ }^{1}$ \\ ${ }^{1}$ School of Architectural and Surveying and Mapping Engineering, Jiangxi University of Science and Technology, Ganzhou 341000, \\ China \\ ${ }^{2}$ Society and Design Faculty, Bond University, Robina QLD 4226, Australia
}

Received 3 November 2019; Accepted 10 February 2020

\begin{abstract}
Phthalate esters (phthalic acid esters or PAEs) are widely utilized during production as an inactive plasticizer, which results in serious water pollution. Ideal removal of micropollutants is difficult to achieve with traditional water treatment, but technology using nanofiltration membrane removes water micropollutants effectively owing to its unique membrane structure. To further study the rejection performance of nanofiltration membrane for PAEs, carbon nanotube-modified nanofiltration membrane was proposed to treat PAEs in water. The adsorption and rejection properties of PAEs on the surface of nanofiltration membrane were revealed by multi-factor experiments. Adsorption kinetics was also analyzed, and the adsorption isotherm of PAEs on the membrane surface was simulated. In addition, the effects of running time, molecular weight, n-octanol/water partition coefficient, and average polarizability on the performance of PAE rejection of nanofiltration membrane were discussed. Results show that the Freundlich adsorption equation simulates the adsorption of PAEs on the surface of the nanofiltration membrane, and the correlation coefficient is more than 0.998. The adsorption capacity of the nanofiltration membrane for three typical PAEs substances, namely, diethyl phthalate (DEP), dibutyl phthalate (DBP), and dioctyl phthalate (DOP), decreases in turn. At the same time, the early rejection of PAEs by nanofiltration membrane is primarily due to the adsorption of membrane surface, and the equilibrium stage depends mainly on the sieving effect of the membrane pores. This study provides a theoretical basis for the application of nanofiltration membrane in the removal of PAEs.
\end{abstract}

Keywords: Nanofiltration membrane, Adsorption, Phthalic acid esters, Organic pollutants

\section{Introduction}

Phthalic acid esters (PAEs), which represent a class of synthetic organic compounds that are mainly used as plasticizers, are added to plastics to obtain better elasticity, durability, and transparency [1]. With the extensive use of plastic products, PAE pollutants are released into the environment. After entering the water, they will transfer to sediments, suspended particles, and organisms through phase distribution, adsorption, and other forms of water flow [2-4]. The physical and chemical properties of PAEs are relatively stable, so they can exist for a long time after entering an organism and can be enriched in the body [5]. They can consequently cause disorders of the human reproductive system and malignant lesions of organs and cells, which will reduce the immune function of the human body [5].

If dissolved PAE pollutants are in drinking water sources, then they will enter into users' drinking water through waterworks. The conventional process in waterworks, which involves coagulation, sedimentation, filtration, and disinfection, can effectively reduce water turbidity and remove particle impurities, bacteria, and viruses. However, the removal of PAE pollutants is poor. Data show that the removal rate of organic matter by conventional water-making process is low. Moreover, PAEs are adsorbed by sediment in the sedimentation tank after

\footnotetext{
*E-mail address: lixind@163.com

ISSN: 1791-2377 @ 2020 School of Science, IHU. All rights reserved.

doi:10.25103/jestr.131.06
}

treatment and then slowly released, resulting in an increase in the content of PAEs in the effluent.

In existing studies, scholars have performed experimental exploration on the treatment of PAEs in water. Many waterworks have added advanced water treatment processes, such as activated carbon adsorption [6], advanced oxidation [7], and membrane separation [10] to ensure effluent quality. The problem is that most of the research results cannot be applied due to material or technical reasons. The performance of nanofiltration membrane to intercept these micropollutants must be explored and the pollutant removal mechanism should be determined. Such an investigation is important to provide theoretical basis for the practical application of the nanofiltration membrane technology.

Modified nanofiltration membrane with nanometer pore size was selected to achieve the objectives of this research. This work aims to study the interception of PAEs, investigate the adsorption and rejection performance of PAEs on the surface of nanofiltration membrane, determine the influence of related factors on PAEs, and explore the removal mechanism of PAEs via nanofiltration membrane. By meeting this objective, this research intends to provide theoretical reference for the application of nanofiltration membrane technology in the removal of PAEs.

\section{State of the art}

At present, the main methods to remove PAEs from water include biodegradation [8], advanced oxidation [9], and 
membrane separation [10]. Chi et al. [11] selected three species of marine microalgae to evaluate the tolerance and biodegradation of two typical PAEs (diethyl phthalate (DEP) and dibutyl phthalate (DBP)). Benthic diatoms have strong adaptability and biodegradation to PAEs. However, when multiple PAEs coexist in one system, antagonism occurs among microorganisms, resulting in a decline in biodegradation. $\mathrm{Li}$ et al. [12] biodegraded diethylhexyl phthalate (DEPH) by short rod gram-negative bacteria Burkholderia pyrrocinia B1213 isolated from soil, and they analyzed the degradation products. They then proposed a novel and complete biodegradation pathway. However, due to the complexity of the process and the production of possible carcinogenic metabolites, biodegradation theory warrants further exploration and improvement. Falås et al. [13] used carriers and microorganisms extracted from activated sludge from sewage treatment plants to study the degradation of four kinds of organic micropollutants. They observed that microbial degradation is highly specific, and the water quality of sewage treatment plants is more complex. They also noted a difference in the conversion rate between compound diversity and microbial community specificity, which is one of the reasons biodegradation technologies, has not been applied yet. Dong et al. [14] successfully synthesized an iron-cerium bimetallic catalyst and employed it to activate sodium persulfate to degrade PAEs in marine sediments. The composite has high degradation performance for PAEs, revealing that the catalyst's efficiency in treating sediments containing PAEs. During this process, sulfate radical plays a major role, but the stability and recyclability of the catalyst must be further studied. Mansouri et al. [15] made a comparative study on the removal of DEP from water by several advanced oxidation technologies commonly used. The results showed that DEP was mainly degraded by hydroxyl radicals. However, under different process conditions, the degradation efficiency of DEP by single process was quite different from that by combined process. Various factors must then be considered comprehensively to determine the most suitable oxidation method. Technologies such as biodegradation and advanced oxidation cannot be employed well to practical engineering because of their own defects. By contrast, membrane separation technology (especially nanofiltration technology) has become one of the main technologies for advanced treatment of drinking water due to its unique advantages in the separation process. The pore size of the nanofiltration membrane is between $0.2 \mathrm{~nm}$ and $2.0 \mathrm{~nm}$, which can effectively intercept the organic matter with a relative molecular weight of $150-1000$ as the relative molecular weight of most PAEs is in the middle. Moreover, because of its Donnan effect, nanofiltration membrane can be utilized to effectively remove high valence ions in water.

Numerous studies have demonstrated that nanofiltration membrane separation technology can effectively remove PAEs from water [16-17]. Zhu et al. [18] successfully prepared a new composite membrane with filtration and adsorption properties, with polyvinylidene fluoride (PVDF) as base membrane. The removal rate of BPA is more than $80 \%$ within the contact time of $1.3 \mathrm{~s}$ when the pressure is 0.5 $\mathrm{MPa}$, and the reuse rate of the membrane is high. However, membrane preparation is complex, and automatic control is difficult to achieve. Wang et al. [19] treated three typical PAEs using the combined process of activated carbon (AC) and nanofiltration membrane (NF). The results demonstrated that the removal rates of three typical PAEs by AC-NF process were higher than $99 \%$. Kim et al. [20] chemically modified nanofiltration membrane through graft polymerization and cross-linking. The rejection rate of endocrine disrupting chemicals (EDCs)and phAc of the modified nanofiltration membrane was greatly improved compared with that of the original membrane. Nanofiltration membrane showed excellent rejection performance for PAEs. Most studies have focused on the improvement of the permeation flux and rejection performance of nanofiltration membrane, but few have reported on its rejection mechanism. To efficiently remove PAEs with different physical and chemical properties under varying operating conditions, the mechanisms involved must be deeply studied. The mechanisms of nanofiltration membrane to remove organic micropollutants usually consisted of electrostatic interaction, hydrophobic interaction, and steric hindrance [21]. Licona et al. [22] studied the effect of surface properties of nanofiltration membrane on the rejection performance of many kinds of phAc under different $\mathrm{pH}$ value conditions. They found that the rejection performance of nanofiltration membrane was affected by many factors, and this effect was the result of the interaction of many kinds of forces. Wei et al. [23] discussed the adsorption kinetics of five kinds of PAEs in water with hollow fiber nanofiltration membrane made in the laboratory. Owing to the greater hydrophobic adsorption and steric hindrance of the hollow fiber nanofiltration membrane, the adsorption of PAEs molecules with larger molecular weight reaches saturation for a longer time, and the rejection rate of PAEs was higher. Nonetheless, the mechanism was not deeply studied. Wang [24] found that the rejection rate of PAEs by nanofiltration membrane changed very rapidly at the initial stage, and after a short rapid increase, the rate slowed down until it reached a certain stable value. This phenomenon was also explained by Yoon [25] and Kiso [26] among others. The sharp change in the rejection rate of nanofiltration membrane at the initial stage was due to the adsorption of the membrane surface of the nanofiltration membrane. The initial adsorption depended on the hydrophilicity and hydrophobicity of the separated substances.

Various methods are utilized to study the removal efficiency and rejection performance of PAEs in water in the aforementioned research. However, few studies have been conducted on the adsorption of PAEs by nanofiltration membrane, especially the dynamic adsorption and rejection of PAEs by carbon nanotube (CNT)-modified nanofiltration membrane. In the present study, the continuous dynamic adsorption experiment of nanofiltration membrane was employed to investigate the adsorption kinetic behavior of PAEs on the membrane surface and the fitting of the membrane surface isotherm. The effects of running time, molecular weight (WM), n-octanol/water partition coefficient $(\operatorname{logKow})$, and average polarizability $(\alpha)$ on the performance of PAE rejection of nanofiltration membrane were also studied. The separation mechanism of PAEs by CNT-modified nanofiltration membrane was discussed. The findings provide theoretical reference for the practical application of the removal of PAEs by nanofiltration membrane.

The remainder of this study is organized as follows. The experimental equipment, experimental materials, and test methods used are described in section 3. The adsorption kinetics and isotherm of PAEs on the surface of nanofiltration membrane are studied by adsorption experiments in section 4 . The effects of running time, MW, logKow, and $\alpha$ on the PAE rejection performance of nanofiltration membrane are also discussed. In addition, the 
removal mechanism of PAEs by CNT-modified nanofiltration membrane is analyzed. The study is summarized in the last section, wherein relevant conclusion is also drawn.

\section{Methodology}

\subsection{Test equipment and materials}

The test device is shown in Figure 1.

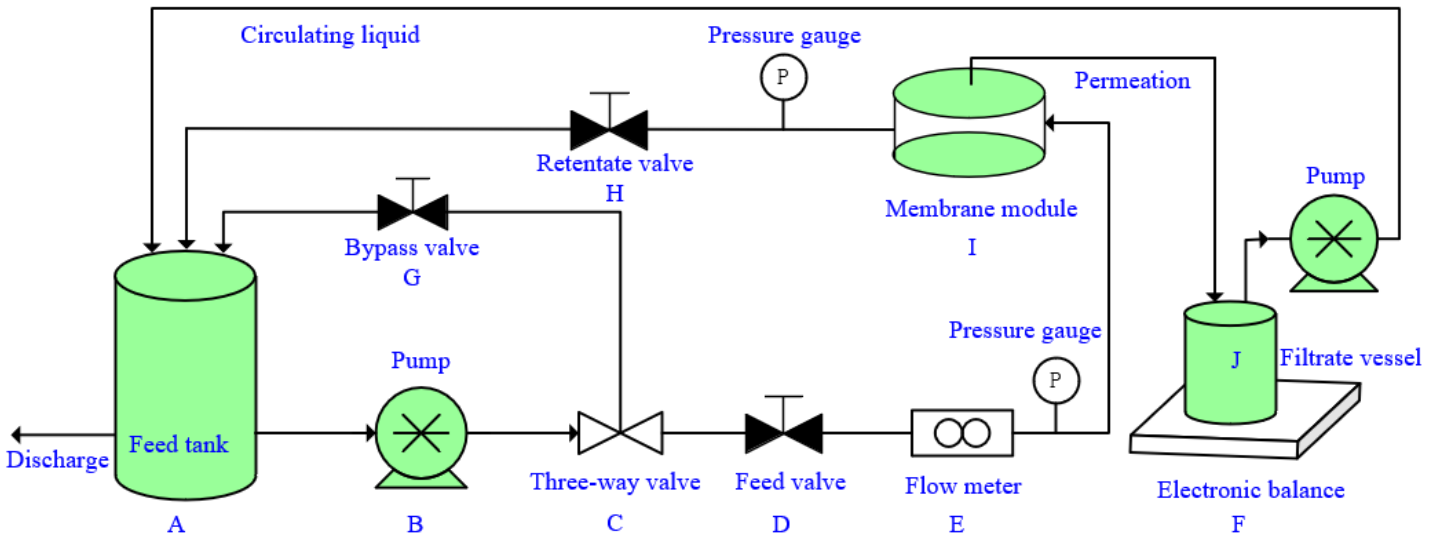

Fig. 1. Schematic of the NF system

The nanofiltration membrane used in the experiment is homemade CNT-modified nanofiltration membrane NF1. The flux of pure water is 36.37 , and the rejection rate of bivalent ions is $97.6 \%$. The three typical phthalate esters (DEP, DBP, and DOP) are simulated configurations.

The agents used in the test are presented in Table 1.

Table 1. Main medicament for testing

\begin{tabular}{l|l|l|l}
\hline Pharmaceutical name & Molecular Formula & Specification & Manufacturer \\
\hline Hydrochloride & $\mathrm{HCl}$ & AR & Jinan Aochen Chemical Co., Ltd. \\
Sodium hydroxide & $\mathrm{NaOH}$ & AR & Jinan Aochen Chemical Co., Ltd. \\
Sodium hypochlorite & $\mathrm{NaClO}_{3}$ & AR & Jinan Aochen Chemical Co., Ltd. \\
Diethyl phthalate (DEP) & $\mathrm{C}_{12} \mathrm{H}_{14} \mathrm{O}_{4}$ & $\geq 99.5 \%$ & National Standard Material Center \\
Dibutyl phthalate (DBP) & $\mathrm{C}_{16} \mathrm{H}_{22} \mathrm{O}_{4}$ & $\geq 99.5 \%$ & National Standard Material Center \\
Dioctyl phthalate (DOP) & $\mathrm{C}_{24} \mathrm{H}_{38} \mathrm{O}_{4}$ & $\geq 99.5 \%$ & National Standard Material Center \\
\hline
\end{tabular}

\subsection{Test method}

(1) Dynamic adsorption of PAEs by nanofiltration membrane

After the nanofiltration membrane was prepressed, the prepared PAEs solution was passed through the test device at a constant volume flow rate. The membrane adsorption test was also carried out, and a blank test was set up for control.

The adsorption capacity of PAEs in the experimental device was calculated according to the mass conservation relationship.

$C_{0} \times V_{0}=W_{m}+C_{1} \times V_{1}+C_{2} \times V_{2}$

$V_{0}=V_{1}+V_{2}$

In Formula (1) and Formula (2), $C_{0}$ is the concentration of PAEs in the influent of the device, $\mathrm{mg} / L ; C_{1}$ and $C_{2}$ are the concentration of PAEs in the permeate and concentrate collected during the t-time period, $\mathrm{mg} / L ; W_{m}$ is the adsorption capacity of the PAEs of the test device equipped with membrane; $V_{0}$ is the total influent volume in t-time period, $L ; V_{1}$ is the permeate liquid volume collected in t-time period, $L$; and $V_{2}$ is the volume of concentrated liquid collected in t-time period, $L$.

Blank test process:
$C_{3} \times V_{3}=W_{n}+C_{4} \times V_{3}$

In Formula (3), $C_{3}$ is the concentration of PAEs in the influent, $m g / L ; C_{4}$ is the concentration of PAEs in the effluent collected during the t-period of operation, $m g / L$; $V_{3}$ is the total influent (effluent) volume in the t-period of operation, $L$; and $W_{n}$ is the adsorption capacity of PAEs by the unfilmed test device.

Under the condition that the initial concentration and flow rate of influent were the same, the adsorption amount per unit membrane area was calculated according to the adsorption amount of PAEs on the experimental device with and without membrane.

The calculation formula is as follows:

$\Gamma_{m, t}=\frac{W_{m}-W_{n}}{A}$

In Formula (4), $A$ is the effective area of nanofiltration membrane, $m^{2}$ and $\Gamma_{m, t}$ is the adsorption amount of PAEs per unit membrane area at $\mathrm{t}$ time, $\mu \mathrm{g} / \mathrm{m}^{2}$.

(2) Rejection effect of nanofiltration membrane on PAEs The nanofiltration cross flow test was carried out by using the experimental device shown in Figure 1. From the beginning of the experiment to the equilibrium of adsorption on the membrane surface, the water samples were taken 
every $10 \mathrm{~min}$ to determine the flux of pure water and rejection rate of pollutant. The calculation methods of these two parameters are as follows:

$$
\begin{aligned}
& J=\frac{V_{P}}{A \cdot t} \\
& R=\left(1-\frac{\rho_{p}}{\rho_{f}}\right) \times 100 \%
\end{aligned}
$$

In Formula (5) and Formula (6), $J$ is the flux, $L /\left(m^{2} \cdot h\right) ; V_{P}$ is volume of permeate solution, $A$ is the effective area of nanofiltration membrane, $\mathrm{m}^{2} ; t$ is running time, $\min ; R$ is the rejection rate, $\% ; \rho_{f}$ is the mass concentration of PAEs in raw water, $m g / L$; and $\rho_{p}$ is the mass concentration of PAEs in permeate, $m g / L$.

\section{Result Analysis and Discussion}

\subsection{Adsorption kinetics of PAEs on the surface of nanofiltration membrane}

Based on the dynamic adsorption experiment of PAEs by nanofiltration membrane in 3.2 , the adsorption kinetics of PAEs on the surface of nanofiltration membrane was analyzed at $0.6 \mathrm{MPa}$ and room temperature when the initial concentration was $200 \mu g / L, 500 \mu g / L$, and $800 \mu g / L$. The adsorption kinetics curve is demonstrated in Figure 2.

Figure 2 shows that the adsorption capacity on the surface of nanofiltration membrane increases with the increase of the initial concentration of PAEs. The adsorption of PAEs on the surface of nanofiltration membrane can be divided into the rapid and slow adsorption stages. In the rapid adsorption stage, the adsorption amount of PAEs on the surface of nanofiltration membrane is approximately linear with time. After the rapid adsorption in the early stage, the adsorption rate slows down because the concentration of PAEs in the solution is much higher than that on the membrane surface. PAEs will then rapidly spread to the membrane surface. With the continuous diffusion and membrane surface adsorption, the difference between the concentration of PAEs in the solution and the concentration on the surface of the nanofiltration membrane decreases, thus entering the slow adsorption stage. With the continuous accumulation of PAEs on the surface of nanofiltration membrane, some of the PAEs adsorbed on the membrane surface will release and fall off simultaneously. Finally, PAEs tend to reach equilibrium with the decrease of the adsorption/release ratio.

Figure 2 also indicates that the initial concentration of PAEs greatly influence the time of adsorption equilibrium on the surface of nanofiltration membrane. In Figure 2(a), when the initial concentration of PAEs is low, the time required for adsorption to reach equilibrium is shorter. This is because PAEs are easily adsorbed by negatively charged nanofiltration membrane. When the concentration is low, PAEs will be adsorbed on the membrane surface, even into the membrane pore. At high concentration, a large number of positively charged PAEs will exhibit strong repulsive crowding when moving to the surface of nanofiltration membrane, reaching the adsorption equilibrium later than at low concentration.

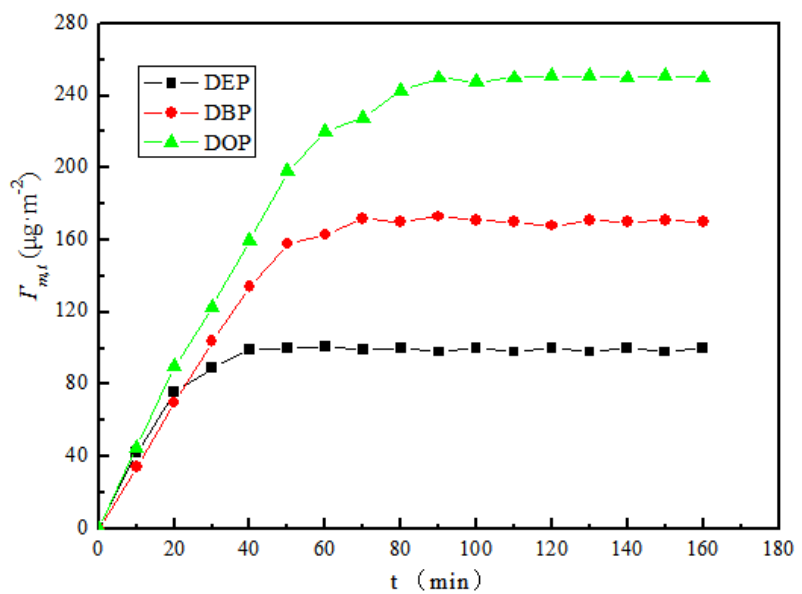

(a) $200 \mu g / L$

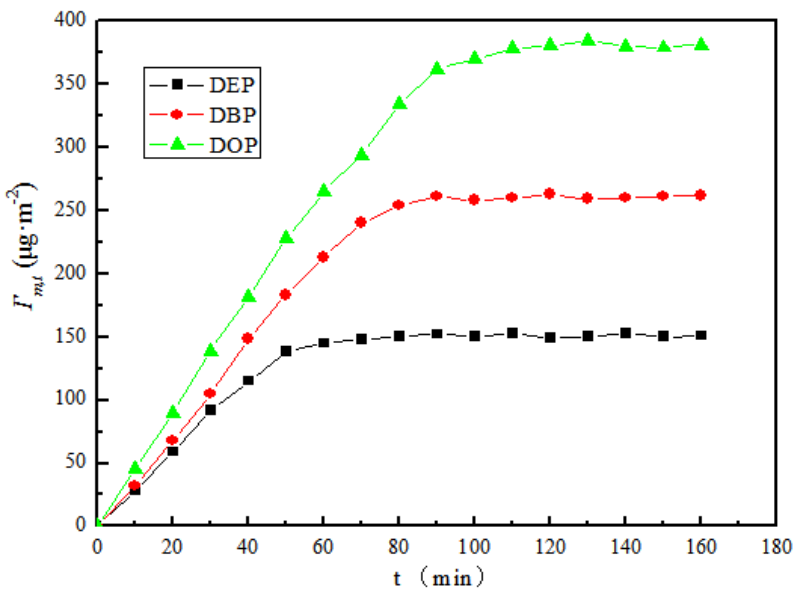

(b) $500 \mu g / L$

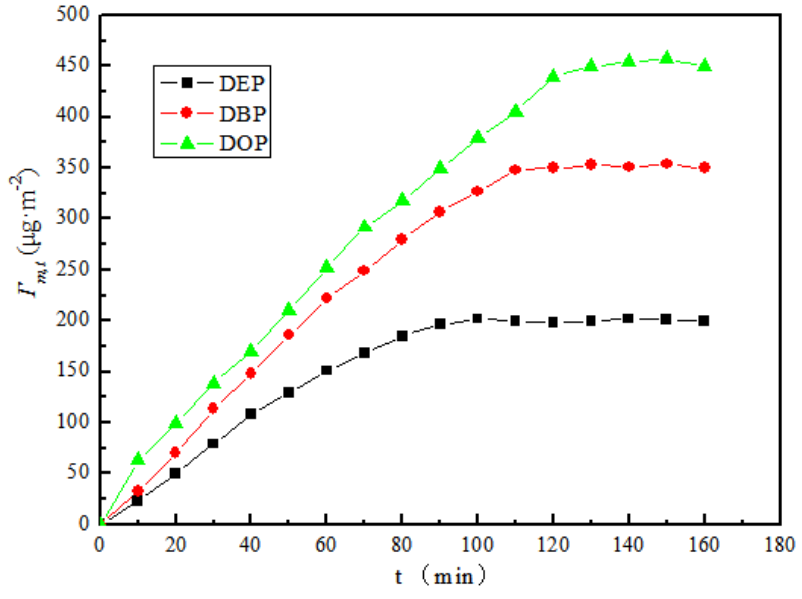

(c) $800 \mu g / L$

Fig. 2. Adsorption kinetics curve of PAEs on the surface of nanofiltration membrane at different initial concentrations

\subsection{Adsorption isotherm of PAEs on the surface of nanofiltration membrane}

Using the data from the tests on the modified nanofiltration membrane's adsorption and rejection of PAEs as basis, the adsorption isotherm of PAEs on the membrane surface was analyzed. The adsorption isotherms of PAEs on the surface of nanofiltration membrane were fitted by Langmuir and Freundlich equation [27]. The fitted parameters are listed in Table 2, and the corresponding fitted adsorption isotherms are presented in Figure 3. 
Table 2. Fitting parameters corresponding to Langmuir and Freundlich adsorption equations

\begin{tabular}{|c|c|c|c|c|c|c|}
\hline Fitting model & \multicolumn{3}{|c|}{ Langmuir } & \multicolumn{3}{|c|}{ Freundlich } \\
\hline PAEs & $\boldsymbol{Q}_{d}\left(\mu g / m^{2}\right)$ & $\boldsymbol{b} /(L / \mu g)$ & $R^{2}$ & $\boldsymbol{K}$ & $1 / n$ & $R^{2}$ \\
\hline DEP & 456.4 & 0.001264 & 0.9852 & 0.0537 & 0.6203 & 0.9986 \\
\hline DBP & 748.6 & 0.001248 & 0.9756 & 0.0914 & 0.6203 & 0.9988 \\
\hline DOP & 1047.7 & 0.001331 & 0.9814 & 0.1688 & 0.5876 & 0.9987 \\
\hline
\end{tabular}

Table 2 reveals that the two equations can fit well the adsorption behavior of PAEs on the surface of nanofiltration membrane. The fitting result of Freundlich isotherm is more stable and reliable, and the correlation coefficient $\mathrm{R} 2$ is more than 0.998. This is because the surface of nanofiltration membrane is heterogeneous and is more suitable for Freundlich adsorption equation. In the Freundlich adsorption isotherm, the size of the adsorption capacity index $1 / n$ reflects the degree to which the surface adsorption capacity of the nanofiltration membrane increases with the concentration [28]. In general, if $0.1<1 / \mathrm{n}<0.5$, then PAEs are easily adsorbed by nanofiltration membrane. If $1 / \mathrm{n}>2$, then PAEs are difficult to be adsorbed by nanofiltration membrane. As $1 / \mathrm{n}$ of DEP, DBP, and DOP are all approximately 0.6 , these three kinds of PAEs are easily adsorbed by nanofiltration membrane. The adsorption constant $\mathrm{K}$ in Freundlich adsorption isotherm indicates the adsorption capacity of nanofiltration membrane. The $\mathrm{K}$ value increases in turn, exhibiting that the adsorption capacity of nanofiltration membrane for DEP, DBP, and DOP decreases in turn.

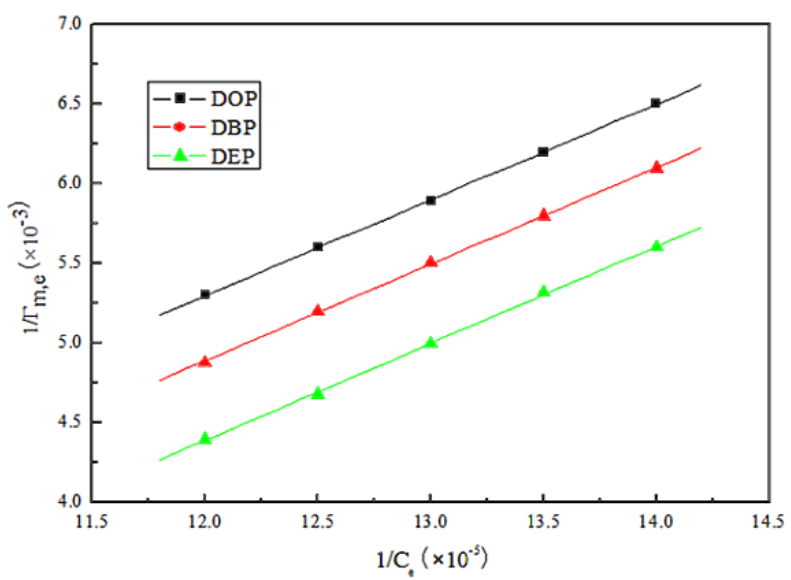

(a)

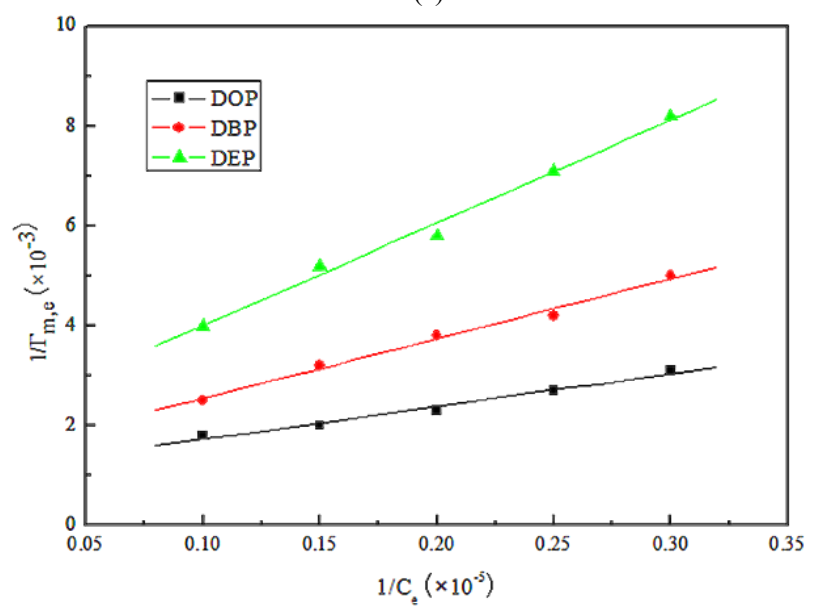

(b)

Fig. 3. Adsorption isotherm of PAEs by nanofiltration membrane. (a) Langmuir adsorption isotherm, (b) Freundlich adsorption isotherm.
4.3 Effect of running time on PAE rejection by nanofiltration membrane

Based on the test on the PAE rejection performance of nanofiltration membrane, the rejection of PAEs by nanofiltration membrane after running continuously for 360 min at room temperature was analyzed when the operating pressure was $0.6 \mathrm{MPa}$ and the initial concentration was 50 min (Figure 4). Figure 4 (a) shows that the water flux of nanofiltration membrane to PAEs decreases with the increase of running time and decreases more and more slowly in the later stage until it stabilizes at a certain value. Figure 4 (b) illustrates that the PAE rejection rate of nanofiltration membrane increases at the initial stage of operation and begins to decrease slowly until it reaches equilibrium at nearly $240 \mathrm{~min}$. According to the fine pore model of nanofiltration membrane [29], the rejection of organic pollutants by nanofiltration membrane can be divided into two steps. (1) Pollutants are adsorbed to the surface of the membrane, and (2) pollutants are converted or diffused through the membrane. Therefore, after running for a certain period, part of the PAEs adsorbed on the membrane surface will pass through the membrane through dissolution and diffusion, resulting in a decrease in the rejection rate. Finally, the dissolved PAEs and adsorbed PAEs on the membrane surface reach a dynamic equilibrium, and the rejection rate tends to have a stable value. The findings are consistent with the results of Yoon [25] and Kiso [26]. That is, the membrane surface adsorption only plays a leading role in the initial stage of filtration. When the membrane surface adsorption reaches saturation, part of the PAEs enriched and adsorbed on the membrane surface will diffuse or even penetrate the membrane. As a result, the rejection effect of the nanofiltration membrane decreases at this point compared with that at the initial stage.

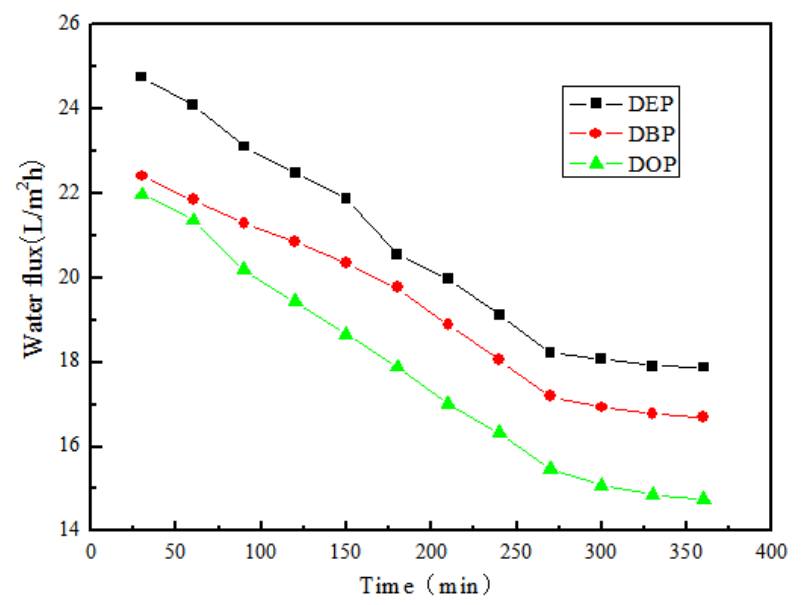

(a) 


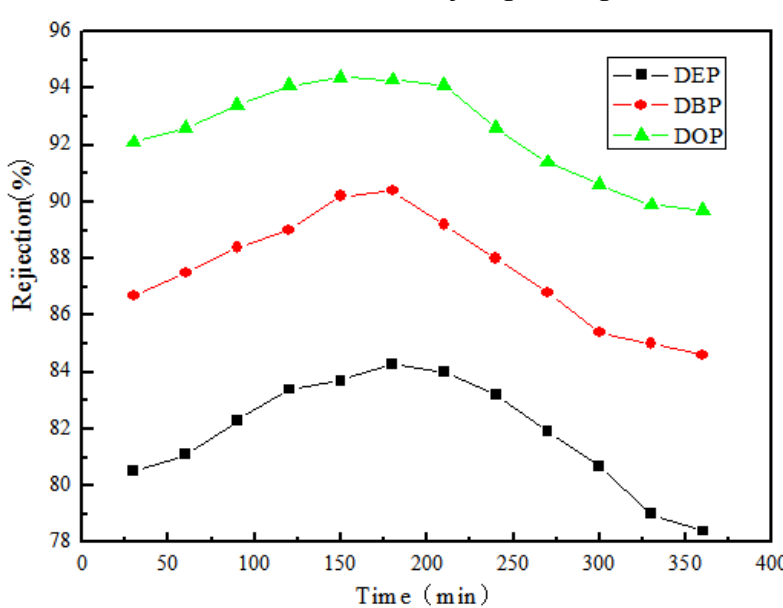

(b)

Fig. 4. Effects of (a) running time on nanofiltration membrane flux effect and (b) running time on nanofiltration membrane rejection

\subsection{Effect of molecular weight on PAE rejection} performance by nanofiltration membrane

From the interception test of PAEs by 3.2 nanofiltration membrane, the effects of WM of DEP, DBP, and DOP on the PAE rejection performance of nanofiltration membrane were determined at room temperature, operating pressure of $0.6 \mathrm{MPa}$ and initial concentration of 50. The experimental results are shown in Figure 5. The molecular weights of DEP, DBP, and DOP are 222.24, 278.34, and 390.55, respectively. The equilibrium rejection rate of nanofiltration membrane increases with the increase of molecular weight of PAEs. This outcome is consistent with the results of Van der Bruggen [30] and Ozaki [31] because the larger the molecular weight of the material, the larger the space volume. Therefore, the greater the steric hindrance, the more difficult for the substance to pass through the membrane pore.

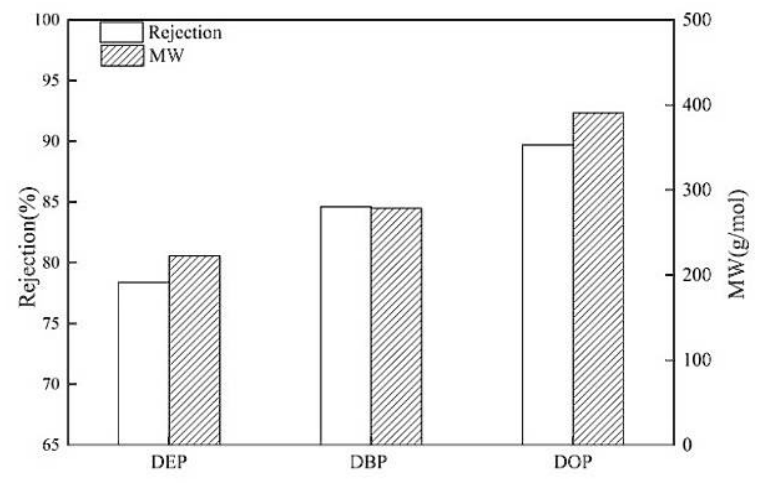

Fig. 5. Effect of WM on PAE rejection performance of nanofiltration membrane

4.5 Effect of n-octanol/water partition coefficient on PAE rejection performance by nanofiltration membrane

Based on the test on the PAE rejection performance of nanofiltration membrane, the effects of logKow of DEP, DBP, and DOP on the PAE rejection performance of nanofiltration membrane were measured at room temperature, operating pressure of $0.6 \mathrm{MPa}$, and initial concentration of 50. The experimental results are shown in Figure 6. The logKow of DEP, DBP, and DOP are 2.52, 4.4, and 8.23 [32], respectively. The higher the logKow value of the substance, the stronger the hydrophobicity. Figure 6 demonstrates that the equilibrium rejection rate of nanofiltration membrane increases with the increase of PAEs' logKow value. This is because the CNT-modified nanofiltration membrane is hydrophilic and has better rejection effect for hydrophobic substances.

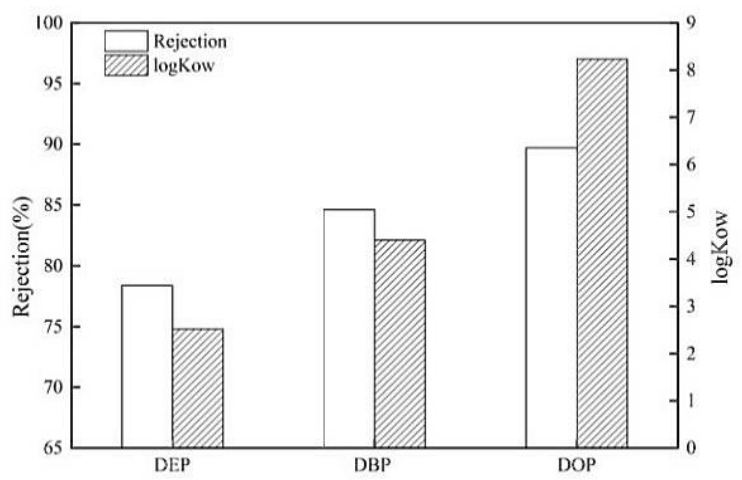

Fig. 6. Effect of logKow on PAE rejection performance of nanofiltration membrane

\subsection{Effect of average polarizability on PAEs rejection by nanofiltration membrane}

Based on the interception test of nanofiltration membrane to PAEs, the effect of $\alpha$ of DEP, DBP, and DOP on the rejection performance of nanofiltration membrane was determined at room temperature, operating pressure of 0.6 $\mathrm{MPa}$, and initial concentration of 50 . The experimental results are shown in Figure 7. $\alpha$ refers to the dipole moment of a molecule induced by a molecule with a dipole nearby, which characterizes the polarity of the molecule. The $\alpha$ values of DEP, DBP, and DOP are 23.41, 30.76, and 45.42, respectively. Figure 7 illustrates that the equilibrium rejection rate of nanofiltration membrane increases with the increase of the $\alpha$ values of PAEs. According to Kaliszan [33], $\alpha$ reflects the volume of molecules to an extent, and the two are approximately proportional to each other. Therefore, the larger the polarizability and space volume of PAEs, the more difficult they are to diffuse through the pore size of the membrane, and the easier they are to be intercepted by the nanofiltration membrane

\section{Conclusions}

To investigate the adsorption and rejection of PAEs by CNT-modified nanofiltration membrane and explore the removal mechanism of PAEs by the modified nanofiltration membrane, small-scale experiments were conducted. The dynamic adsorption process of PAEs on the surface of nanofiltration membrane were investigated, and the effects of running time, WM, $\operatorname{logKow}$ and $\alpha$ on the PAE rejection performance of nanofiltration membrane were determined. The adsorption kinetics of PAEs on the membrane surface and the fitting of the membrane surface isotherm were discussed. The following conclusions are drawn:

(1) The adsorption capacity of PAEs by nanofiltration membrane increases with the increase of initial concentration of raw material, and the adsorption process can be divided into fast and slow adsorption stages. The initial concentration of PAEs greatly influence the adsorption equilibrium time on the surface of nanofiltration 
membrane. When the initial concentration is low, the adsorption equilibrium time is shorter.

(2) The adsorption isotherms of DEP, DBP, and DOP on the surface of nanofiltration membrane were fitted by Langmuir and Freundlich adsorption equations. The Freundlich adsorption equation can better simulate the adsorption of PAEs on the surface of nanofiltration membrane, and the correlation coefficient R2 is more than 0.998 .

(3) In intercepting PAEs, the flux of nanofiltration membrane decreases, but the decline rate becomes slower until it remains stable. With the running time, the rejection rate increases at first, then decreases, and finally reaches a stable value. The results show that the rejection of PAEs by nanofiltration membrane can be divided into two stages. The early stage mainly depends on the adsorption of the membrane surface, and the equilibrium stage mainly depends on the sieving effect of membrane pores.

By combining laboratory experiments and theoretical research, the mechanism of PAE removal by CNT-modified nanofiltration membrane was proposed. Adsorption theory together with the analysis of influencing factors provide a theoretical basis for the application of nanofiltration membrane in PAE removal. As many influencing factors exist in natural water body, natural water body will be used in future research. The mechanism will be further modified with reference to this study to better guide the practical application of membrane technology.

\section{Acknowledgments}

This work was financially supported by the National Natural Science Foundation of China (No. 41662004), Science and Technology Research Project of Jiangxi Provincial Department of Education (No. GJJ160619), and Engineering Research Center for Water Quality Safety and Security in the Ganjiang River Basin. All the experiments demonstrate that the proposed algorithm is effective and promising.

This is an Open Access article distributed under the terms of the Creative Commons Attribution License

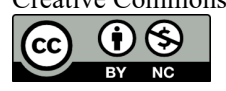

\section{References}

1. Zhang Z M., Zhang H H., Zou Y W., Yang G P., "Distribution and ecotoxicological state of phthalate esters in the sea-surface microlayer, seawater and sediment of the Bohai Sea and the Yellow Sea". Environmental Pollution, 240, 2018, pp. 235-247.

2. Mi L J., Xie Z Y., Zhao Z., Zhong M Y., Ebinghaus R., Tang J H., "Occurrence and spatial distribution of phthalate esters in sediments of the Bohai and yellow seas". Science of The Total Environment, 653,2019 , pp. 792-800.

3. Paluselli A., Aminot Y., Galgani F., Net S., Sempéré R., "Occurrence of phthalate acid esters (PAEs) in the northwestern Mediterranean Sea and the Rhone River". Progress in Oceanography. 163, 2018, pp. 221-231.

4. Wang J., Chen G C., Christie P., Zhang M Y., Luo Y M., Teng Y., "Occurrence and risk assessment of phthalate esters (PAEs) in vegetables and soils of suburban plastic film greenhouses". Science of The Total Environment. 523, 2015, pp. 129-137.

5. Li X D., Zhang M., Dai W C., Kang M H., Zhao B X., "Preservation and migration law of PAEs in the Zhang River of Ganzhou city section". Journal of Jiangxi University of Science and Technology. 38(5), 2017, pp. 51-56.

6. Álvarez P.M., García-Araya J.F., Beltrána F.J., Masa F.J., Medina F., "Ozonation of activated carbons: Effect on the adsorption of selected phenolic compounds from aqueous solutions". Journal of Colloid and Interface Science. 283, 2005, pp. 503-612.

7. Chen P J., Rosenfeldt E J., Kullman S W., Hinton D E., Linden K G., "Biological assessments of a mixture of endocrine disruptors at environmentally relevant concentrations in water following $\mathrm{UV} / \mathrm{H}_{2} \mathrm{O}_{2}$ oxidation". Environmental Science and Technology. 376, 2007, pp. 18-26.

8. Mir-Tutusaus J A., Baccar R., Caminal G., Sarrà M., "Can white-rot fungi be a real wastewater treatment alternative for organic micropollutants removal? A review". Water Research. 138, 2018, pp. 137-151.

9. Lado Ribeiro A R., Moreira N F.F., Puma G L., Silvaa A M.T., "Impact of water matrix on the removal of micropollutants by advanced oxidation technologies". Chemical Engineering Journal. 363, 2019, pp. 155-173.

10. Fudala-Ksiazek S., Pierpaoli M., Luczkiewicz A., "Efficiency of landfill leachate treatment in a MBR/UF system combined with NF, with a special focus on phthalates and bisphenol A removal". Waste Management, 78, 2018, pp. 94-103.

11. Chi J., Li Y., Gao J., "Interaction between three marine microalgae and two phthalate acid esters". Ecotoxicology and Environmental Safety, 170, 2019, pp. 407-411.

12. Li J L., Zhang J F., Yadav M P., Li X T., "Biodegradability and biodegradation pathway of di-(2-ethylhexyl) phthalate by Burkholderia pyrrocinia B1213”. Chemosphere, 225, 2019, pp. $443-450$.
13. Falås P., Jewell K S, Hermes N., Wick A., Ternes T A., Joss A., Nielsend J L., "Transformation, CO2 formation and uptake of four organic micropollutants by carrier-attached microorganisms". Water Research, 141, 2018, pp. 405-416.

14. Dong C D., Huang C P., Nguyen T B., Hsiung C F., Wu C H., Lin Y L., Chen C W., Hung C M., "The degradation of phthalate esters in marine sediments by persulfate over iron-cerium oxide catalyst". Science of The Total Environment, 696, 2019, pp. 443-450.

15. Mansouri L., Tizaoui C., Geissen S U., Bousselmi L., "A comparative study on ozone, hydrogen peroxide and UV based advanced oxidation processes for efficient removal of diethyl phthalate in water". Journal of Hazardous Materials, 363, 2019, pp. 401-411.

16. Dharupaneedi S P., Nataraj S K., Nadagouda M., Reddy K R., Shukla S S., Aminabhavi T M., "Membrane-based separation of potential emerging pollutants". Separation and Purification Technology, 210, 2019, pp. 850-866.

17. Kim S., Chu K H., Al-Hamadania Y A.J., Park C M., Jang M., Kim D H., Yu M., Heo J., Yoon Y., "Removal of contaminants of emerging concern by membranes in water and wastewater: a review". Chemical Engineering Journal, 335, 2018, pp. 896-914.

18. Zhu Y W., Wei J F., Zhang H., Liu K., Kong Z Y., Dong Y., Jin G., Tian J., Qin Z., "Fabrication of composite membrane with adsorption property and its application to the removal of endocrine disrupting compounds during filtration process". Chemical Engineering Journal, 352, 2018, pp. 53-63.

19. Wang L., Wan Q L., Wu J J., Guo M., Mao S., Lin J Q., "Removal of Phthalate Esters by Combination of Activated Carbon with Nanofiltration". Sustainable Development of Water Resources and Hydraulic Engineering in China, 2019, pp. 269-273.

20. Kim J H., Park P K., Lee C H., Kwon H H., "Surface modification of nanofiltration membranes to improve the removal of organic micro-pollutants (EDCs and PhACs) in drinking water treatment: Graft polymerization and cross-linking followed by functional group substitution". Journal of Membrane Science, 321, 2008, pp. 190-198.

21. Bellona C., Drewes J E., Xu P., Amy G., "Factors affecting the rejection of organic solutes during NF/RO treatment--a literature review “. Water Research, 38(12), 2004, pp. 2795-2809.

22. Licona K.P.M., Geaquinto L.R.de O., Nicolini J.V., Figueiredo N.G., Chiapetta S.C., Habert A.C., Yokoyama L., "Assessing potential of nanofiltration and reverse osmosis for removal of toxic pharmaceuticals from water". Journal of Water Process Engineering, 25, 2018, pp. 195-204. 
Xindong Li, Guozi Ouyang, Tingting Tian, Lijinhong Huang, and Xin Zhang/

Journal of Engineering Science and Technology Review 13 (1) (2020) 42 - 49

23. Wei X Z., Shi Y Y., Fei Y W., Chen J Y., Lv B., Chen Y S., Zheng H L., Shen J L., Zhu L P., "Removal of trace phthalate esters from water by thin-film composite nanofiltration hollow fiber membranes". Chemical Engineering Journal, 292, 2016, pp. 382388.

24. Wang L., Dong B Z., Gao N Y., "The effects of issolved organic matters on EDCs (BPA) removal by ultrafiltration". Journal of Shandong Jianzhu University, 24(1), 2009, pp. 9-17.

25. Yoon Y., Westerhoff P., Yoon J., Snyder S A., "Removal of $17 ß$ Estradiol and Fluoranthene by Nanofiltration and Ultrafiltration". Journal of Environmental Engineering, 130(12), 2004, pp. 14601467.

26. Kiso Y., Sugiura Y., Kitao T., Nishimura K., "Effects of hydrophobicity and molecular size on rejection of aromatic pesticides with nanofiltration membranes". Journal of Membrane Science, 192(1-2), 2001, pp. 1-10.

27. Wu D., Du J Y., Li P., "Equilibrium model studies in adsorption of fluoride in aqueous solution: A summary of recent researches". Journal of Nanchang University(Engineering \& Technology), 32(2), 2010, pp. 103-112.
28. Cheng A H., Wang L., Wang X D., Lin F., Zhang L., Zhang R., "Removal of trace phthalate acid esters in water by nanofiltration membrane". Technology of Water Treatment, 33(11), 2007, pp. 1416.

29. Reinhard M, Goodman N L, Mccarty P L, Argo D G., "Removing Trace Organics by Reverse Osmosis Using Cellulose Acetate and Polyamide Membranes". Journal, 78(4), 1986, pp. 163-174.

30. Bruggen B V D., Schaep J., Wilms D., Vandecasteele C., "Influence of molecular size, polarity and charge on the rejection of organic molecules by nanofiltration". Journal of Membrane Science, 156(1), 1999, pp. 29-41.

31. Ozaki H., Li H., "Rejection of organic compounds by ultra-low pressure reverse osmosis membrane". Water Research, 36(1), 2002, pp. 123-30.

32. Long X X., Shi S Q., Niu J F., Shen Z Y., Yang Z F., "The quantum chemical model predicts the octanol water distribution coefficient of phthalate ester". In: The 4th Symposium on Environmental Simulation and Pollution Control, Bei Jing, CHINA, 2005, pp.249250.

33. Kaliszan R., "Quantitative structure-rejection relationships". Analytical Chemistry, 64(11), 2008, pp. 233-246. 